\title{
Defective Phosphorene as a promising anchoring material for lithium-sulfur batteries
}

Hafiz Humza Haseeb ${ }^{\mathrm{a}}$, Yan Li ${ }^{\mathrm{a},}$, Sana Ayub ${ }^{\mathrm{a}}$, Qinglong Fang ${ }^{\mathrm{a}}$, Lijun Yu ${ }^{\mathrm{b}}$, Kewei Xu ${ }^{\mathrm{b}}$, Fei Ma ${ }^{\mathrm{a}}$

${ }^{a}$ State Key Laboratory for Mechanical Behavior of Materials, Xi'an Jiaotong University, Xi'an 710049, Shaanxi, China

${ }^{b}$ Shaanxi Key Laboratory of Surface Engineering and Remanufacturing, Xi'an University, Xi'an 710065, Shaanxi, China 


\section{FIGURE CAPTIONS}

Fig. S1. Optimized structures of (a) $S_{8}$ and (b)-(f) $L i_{2} S_{n}$ (n=1,2,4,6,8). (g) The shortest distances of Li-S and S-S bonds.

Fig. S2. Lowest-energy optimized adsorption configurations of (a)-(e) $L i_{2} S_{x}(x=1,2,4,6,8)$ and (f) $S_{8}$ on $\mathrm{pP}$ substrate, both top view and side view are shown.

Fig. S3 (a) Evaluated binding energies between LiPSs and phosphorene-based substrates (pP, MVP, OP, SP) in DME/DOL (1:1, v:v) electrolyte. (b) Binding energy differences between vacuum systems and corresponding solvent models $\left(\Delta \mathrm{E}=\mathrm{E}_{\mathrm{b}}\left[\right.\right.$ vacuum] $-\mathrm{E}_{\mathrm{b}}[$ solvent $\left.]\right)$.

Fig. S4 Optimized structures and adsorption energies of (a) $\mathrm{Li}_{2} \mathrm{~S}, \mathrm{Li}_{2} \mathrm{~S}_{2}, \mathrm{Li}_{2} \mathrm{~S}_{4}, \mathrm{Li}_{2} \mathrm{~S}_{6}, \mathrm{Li}_{2} \mathrm{~S}_{8}$, and $\mathrm{S}_{8}$, bond with DME, (b) $\mathrm{Li}_{2} \mathrm{~S}, \mathrm{Li}_{2} \mathrm{~S}_{2}, \mathrm{Li}_{2} \mathrm{~S}_{4}, \mathrm{Li}_{2} \mathrm{~S}_{6}, \mathrm{Li}_{2} \mathrm{~S}_{8}$, and $\mathrm{S}_{8}$, bond with DOL.

Fig. S5. Electronic band structures of (a) $L i_{2} S / \mathrm{pP}$, (b) $L i_{2} S_{2} / \mathrm{pP}$, (c) $L i_{2} S_{4} / \mathrm{pP}$, (d) $L i_{2} S_{6} / \mathrm{pP}$ and (e) $L i_{2} S_{8} / \mathrm{pP}$.

Fig. S6. Lowest-energy optimized adsorption configurations of (a)-(e) $L i_{2} S_{x}(x=1,2,4,6,8)$ and (f) $S_{8}$ on OP substrate, both top view and side view are shown.

Fig. S7. Electronic band structures of (a) $L i_{2} S / \mathrm{OP}$, (b) $L i_{2} S_{2} / \mathrm{OP}$, (c) $L i_{2} S_{4} / \mathrm{OP}$, (d) $L i_{2} S_{6} / \mathrm{OP}$ and (e) $\mathrm{Li}_{2} \mathrm{~S}_{8} / \mathrm{OP}$.

Fig. S8. Lowest-energy optimized adsorption configurations of (a)-(e) $L i_{2} S_{x}(x=1,2,4,6,8)$ and (f) $S_{8}$ on SP substrate, both top view and side view are shown.

Fig. S9. Electronic band structures of (a) $L i_{2} S / \mathrm{SP}$, (b) $L i_{2} S_{2} / \mathrm{SP}$, (c) $L i_{2} S_{4} / \mathrm{SP}$, (d) $L i_{2} S_{6} / \mathrm{SP}$ and (e) $\mathrm{Li}_{2} \mathrm{~S}_{8} / \mathrm{SP}$. 
Fig. S10. Atomic orientation of $L i_{2} S$ over (a) pP, (b) OP and (c) SP substrates, while of $L i_{2} S_{8}$ over (d) pP, (e) OP and (f) SP, after the completion of MD simulations.

Fig. S11. Energy evolvement of $L i_{2} S$ and $L i_{2} S_{8}$ over pP, OP and SP structures, as the function of MD calculation time.

Fig. S12. Variances of Li-S $\left(\Delta d_{L i-S}\right)$ and S-S $\left(\Delta d_{S-S}\right)$ bond lengths of LiPSs, after adsorption on pP (black), MVP (red), OP (blue) and SP (magenta). 


\section{FIGURES}

Fig. S1

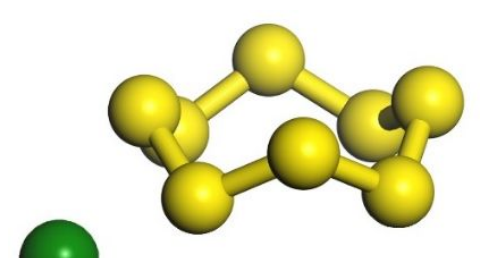

(a)

$\mathrm{Li}$

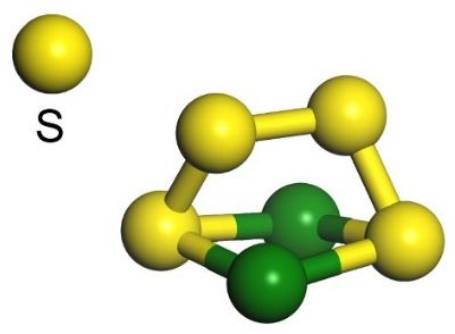

(d)

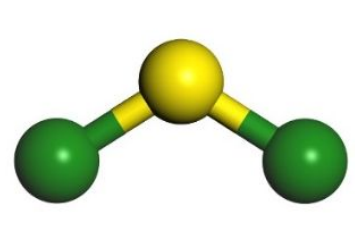

(b)

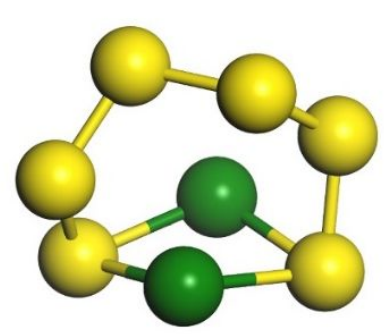

(e)

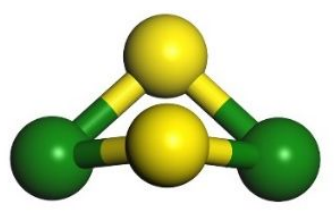

(c)

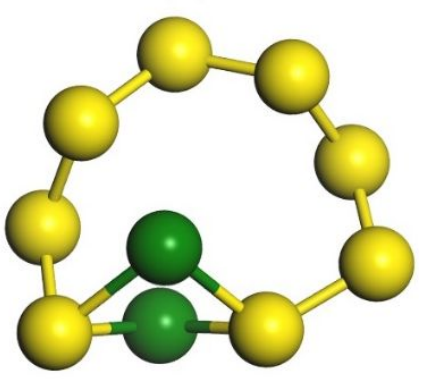

(f)

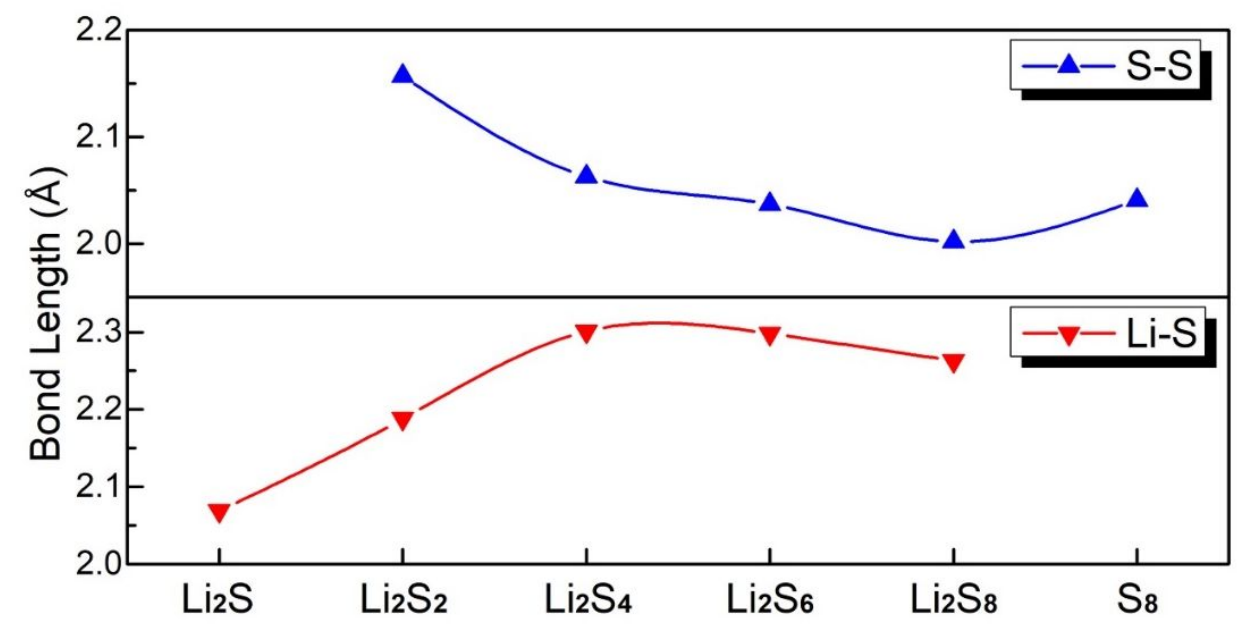

(g) 
Fig. S2
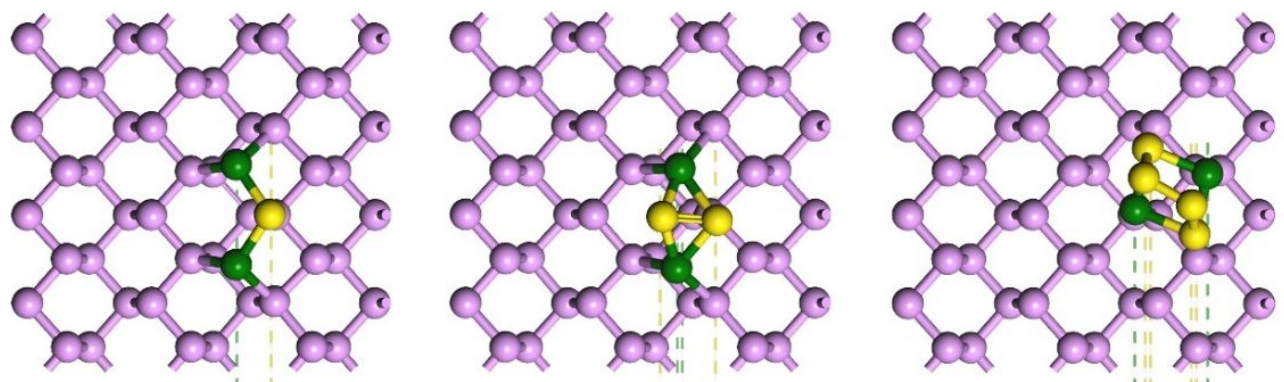

पe colve

(a)
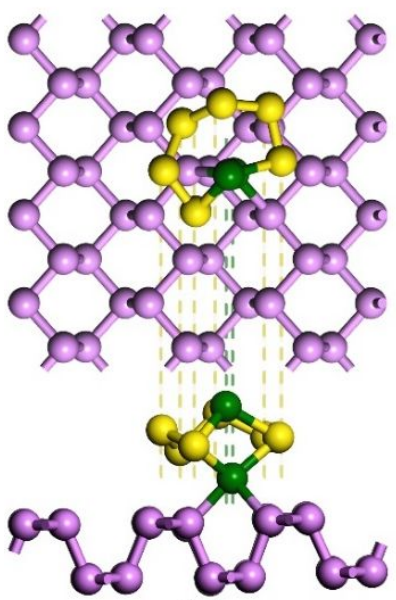

(d)

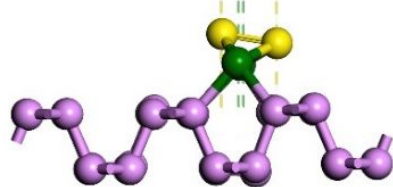

(b)
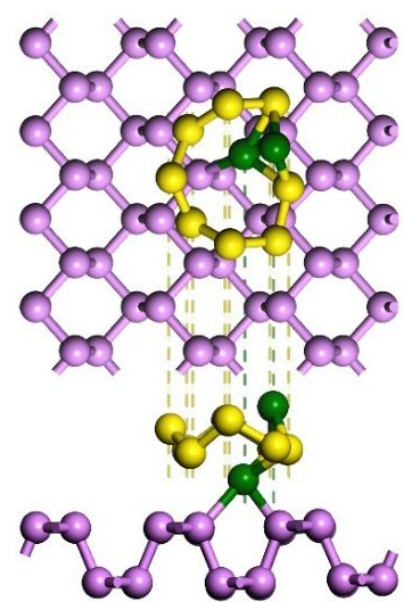

(e)

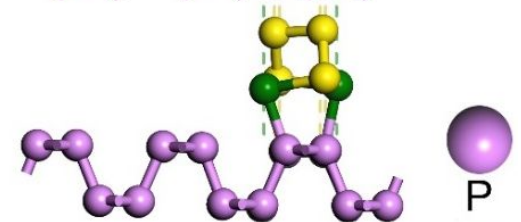

(c)

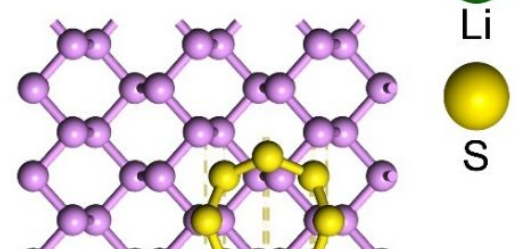

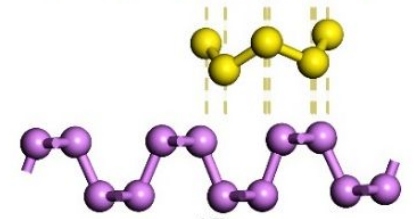

(f) 
Fig. S3
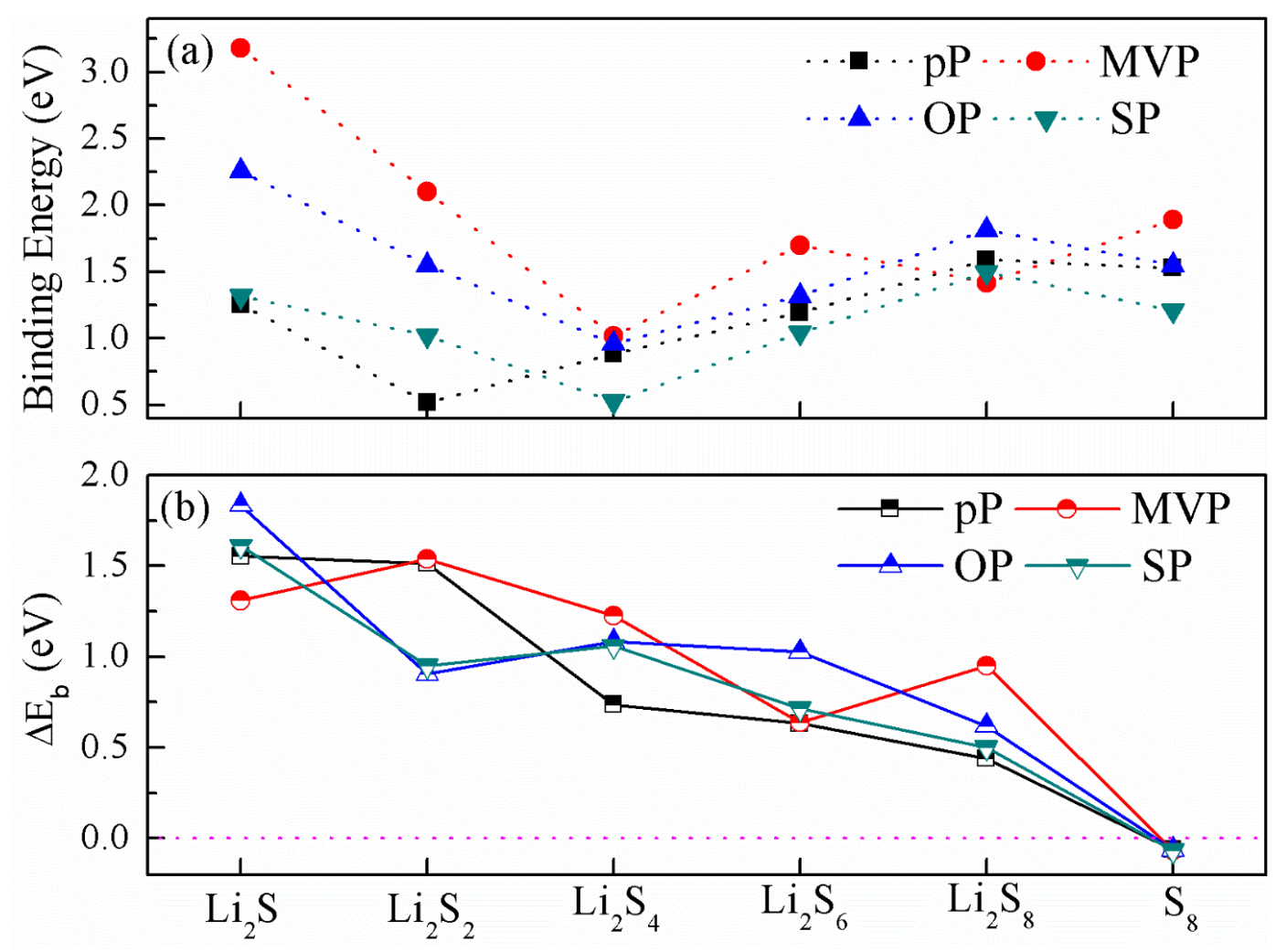

Fig. S4

(a)
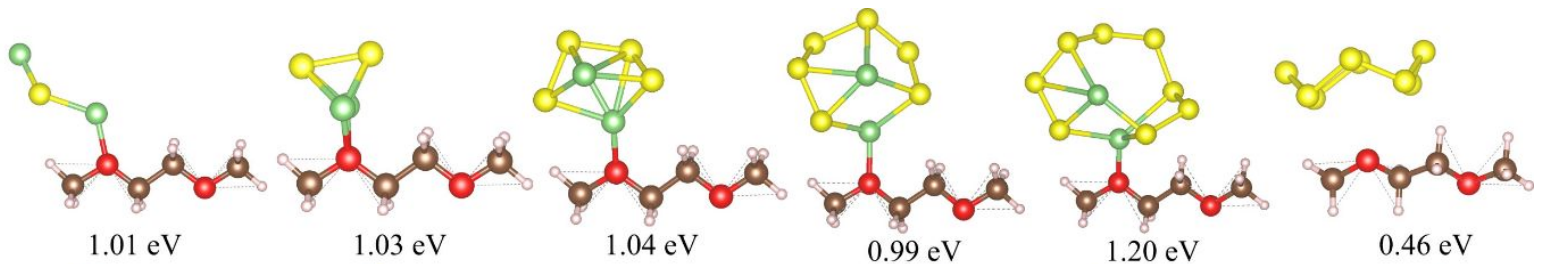

(b)

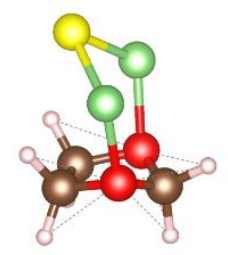

$1.51 \mathrm{eV}$

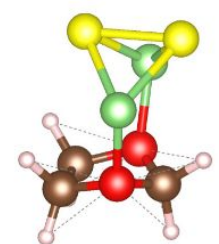

$1.70 \mathrm{eV}$

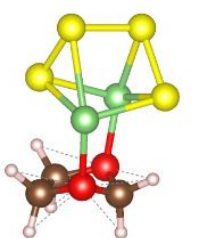

$1.47 \mathrm{eV}$

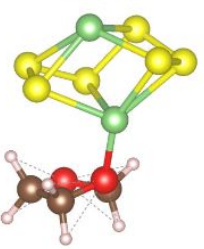

$1.33 \mathrm{eV}$

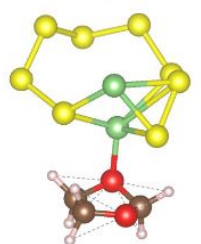

$1.27 \mathrm{eV}$

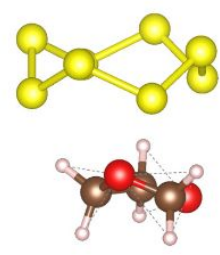

$0.68 \mathrm{eV}$ 
Fig. S5

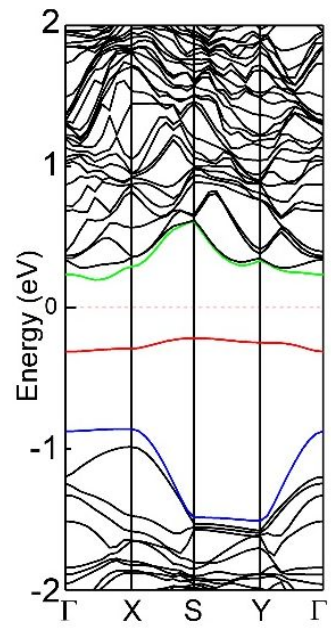

(a)

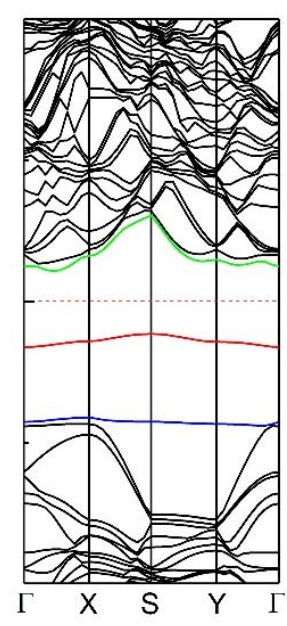

(b)

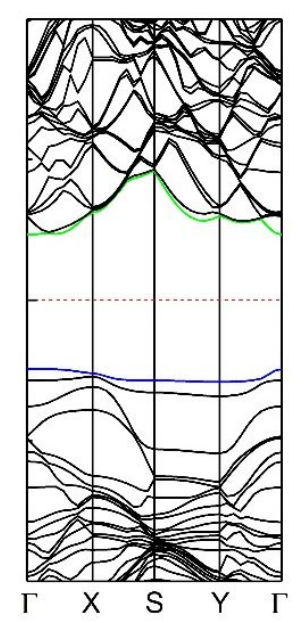

(c)

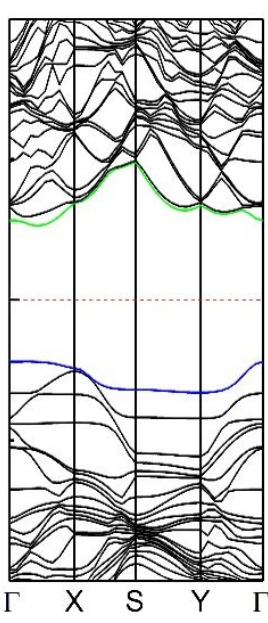

(d)

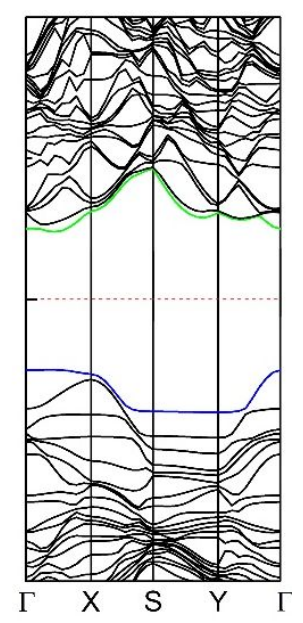

(e) 
Fig. S6
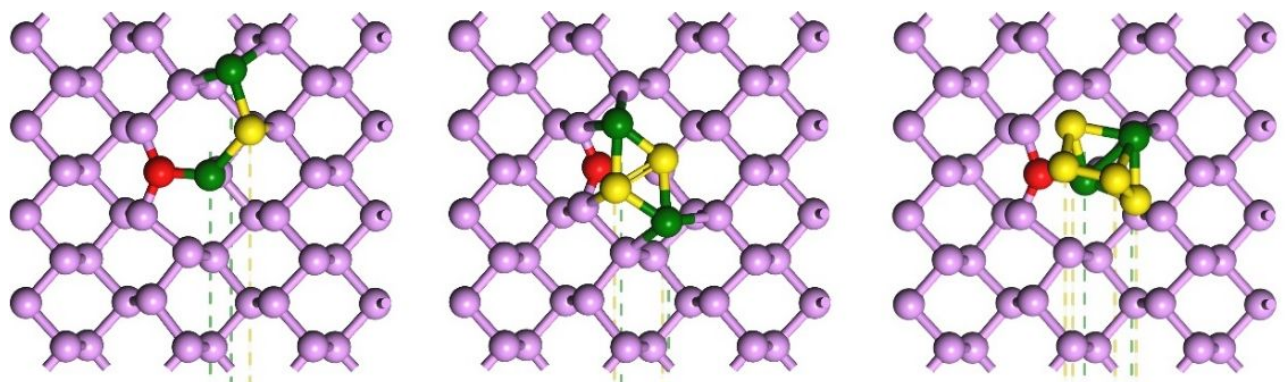

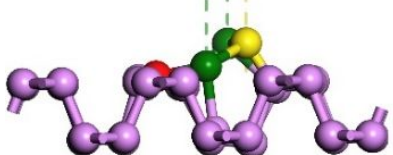

(a)
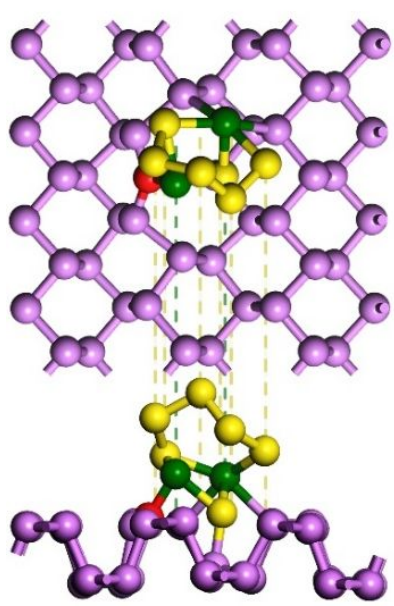

(d)

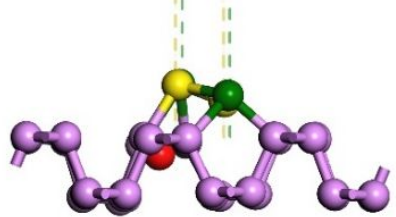

(b)
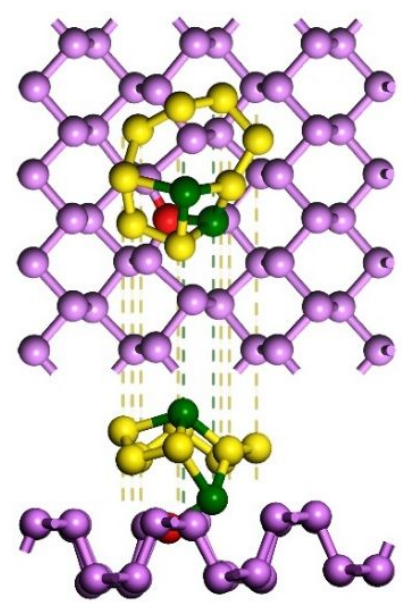

(e)
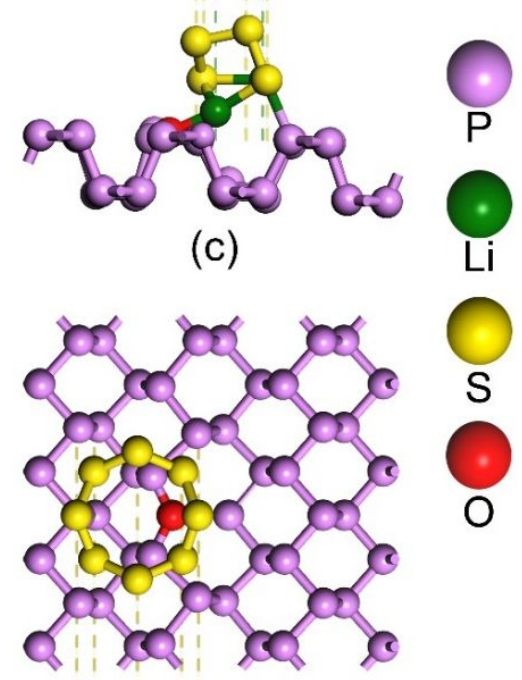

800

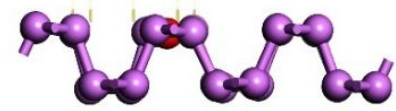

(f) 
Fig. S7

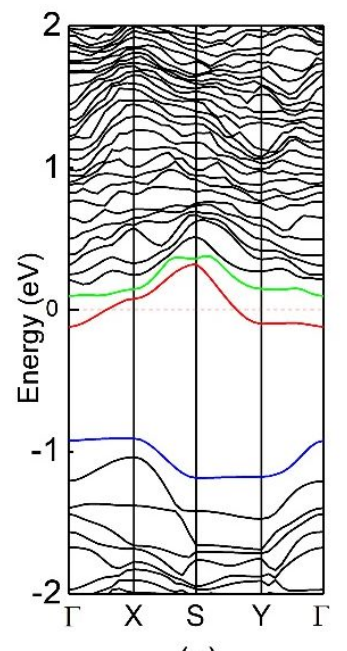

(a)

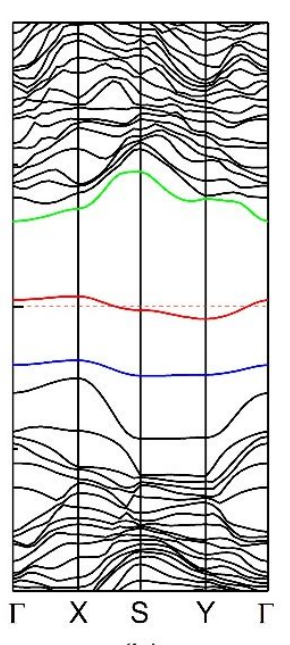

(b)

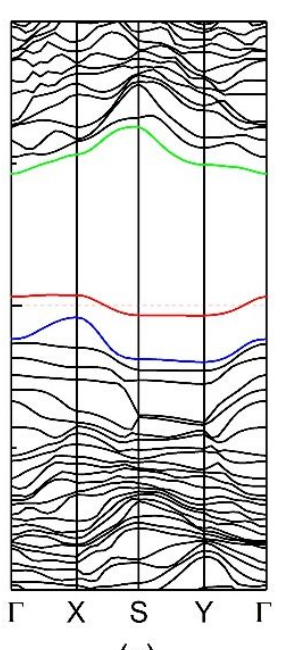

(c)

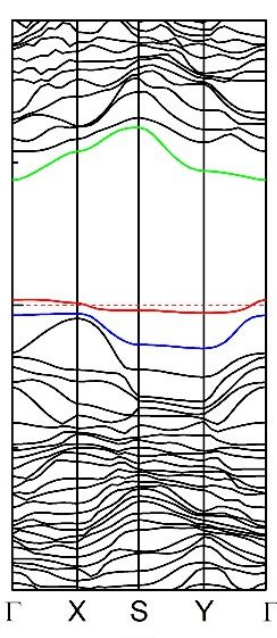

(d)

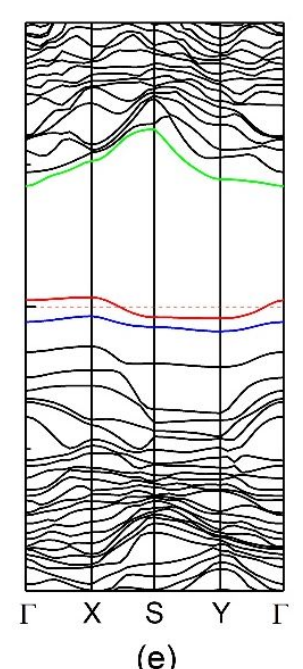

(e) 
Fig. S8

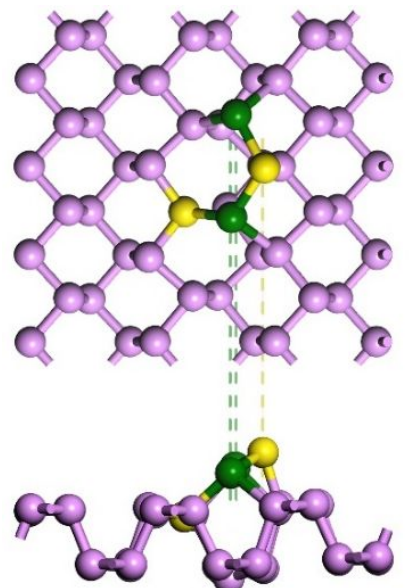

(a)

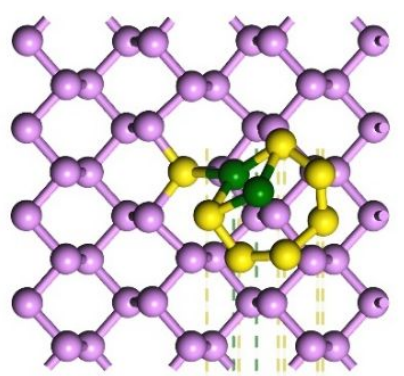

9. 0 bo

(d)

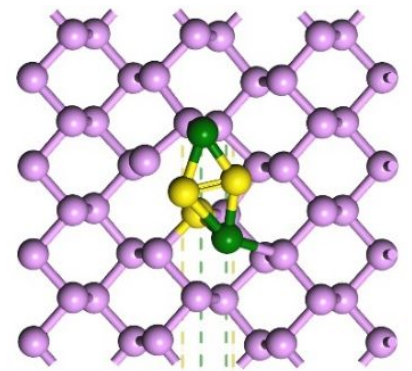

कe refinge

(b)
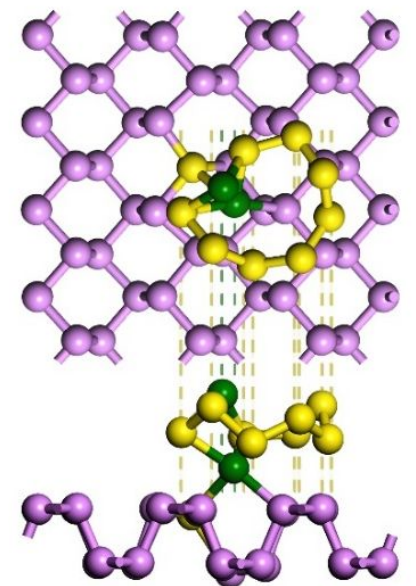

(e)

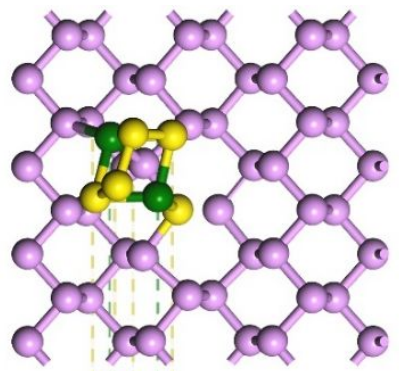

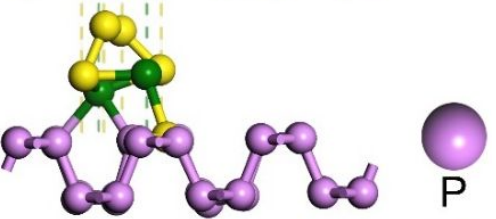

(c)
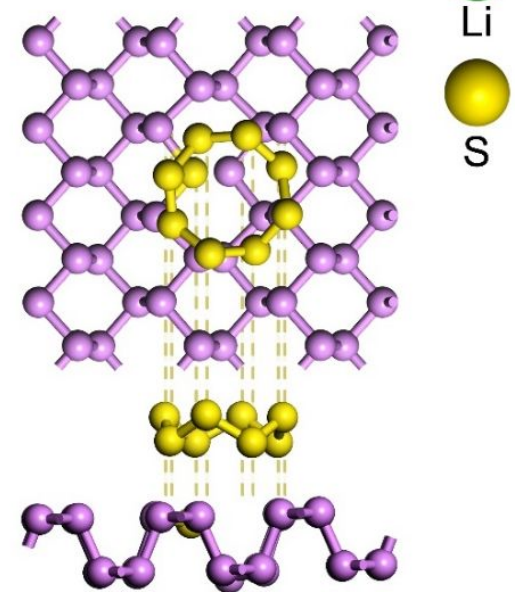

(f) 
Fig. S9

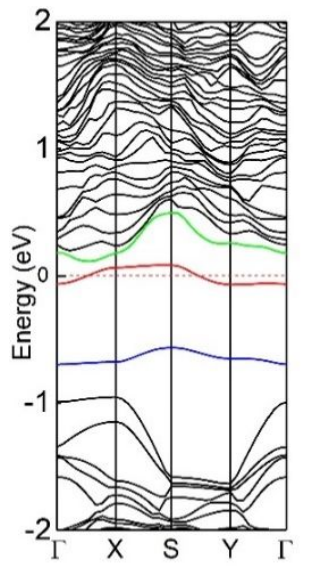

(a)

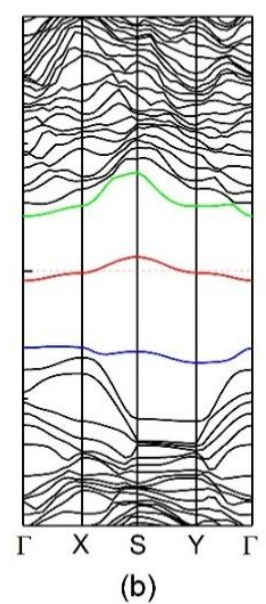

(b)

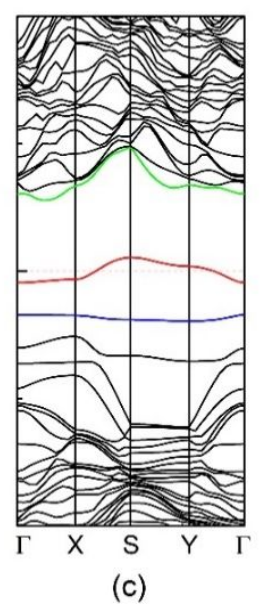

(c)

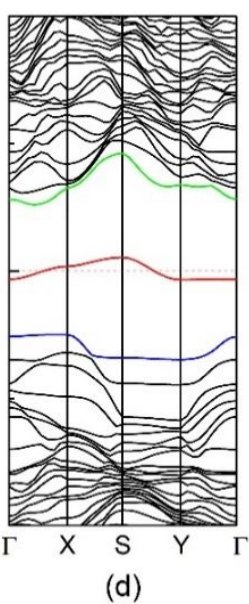

(d)

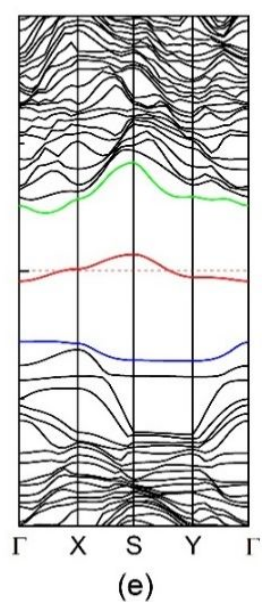

(e) 
Fig. S10
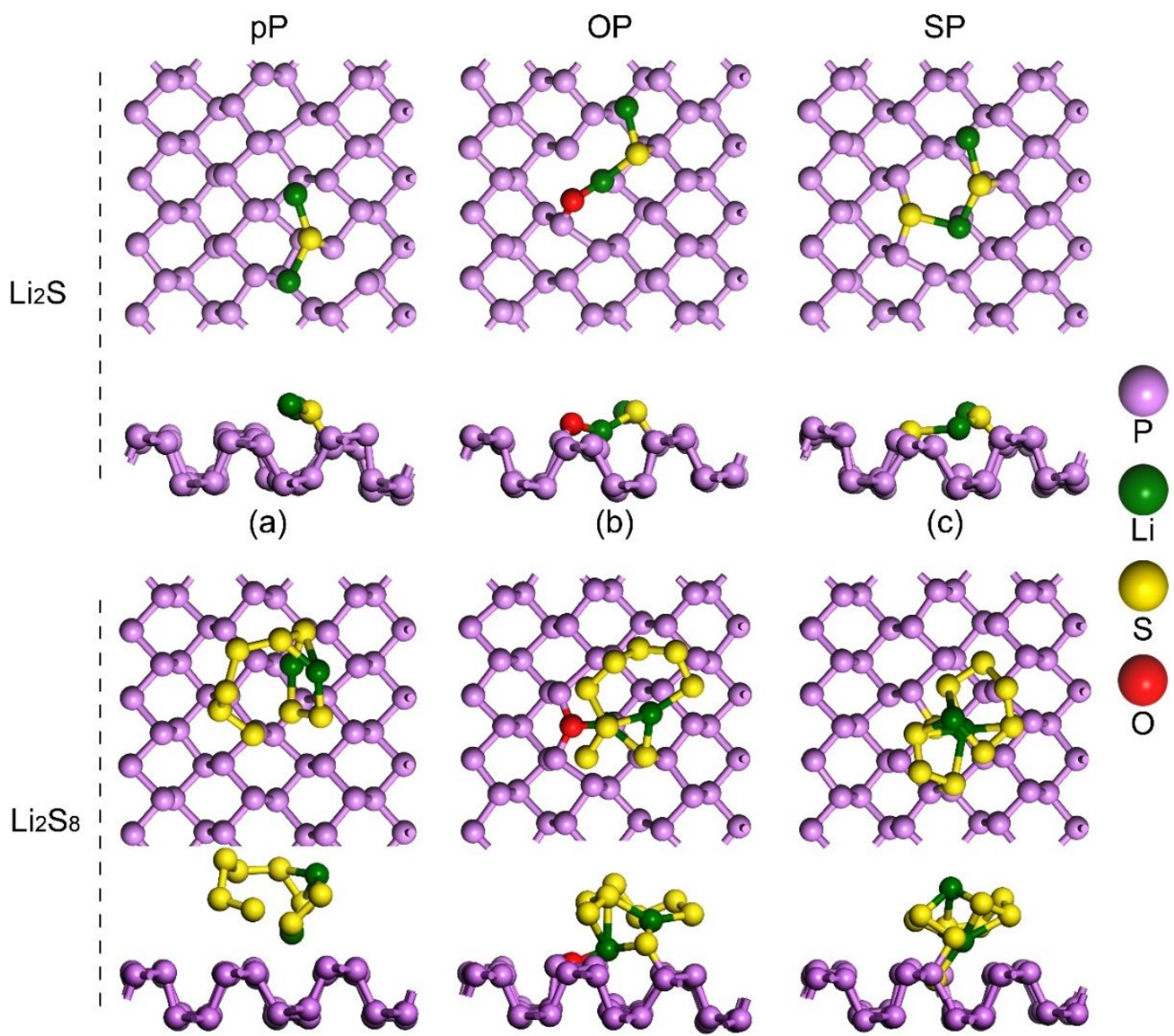

(d)

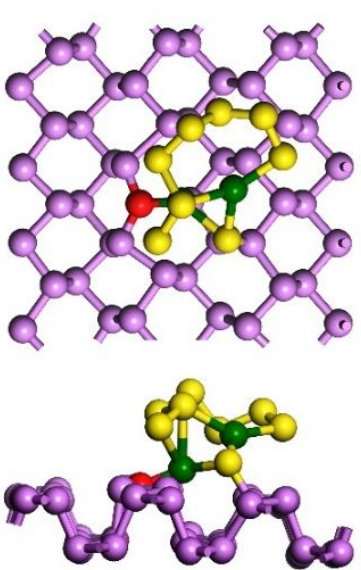

(e)
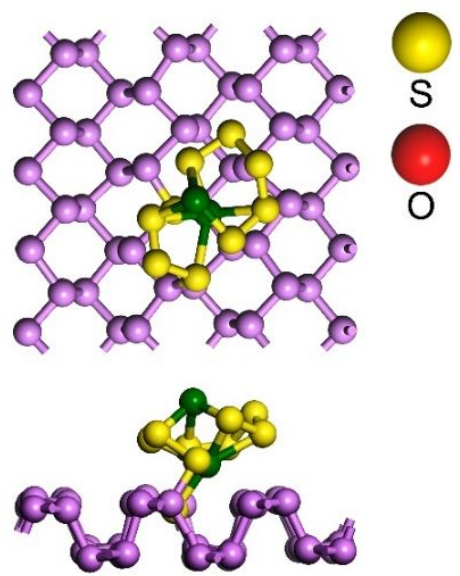

(f) 
Fig. S11
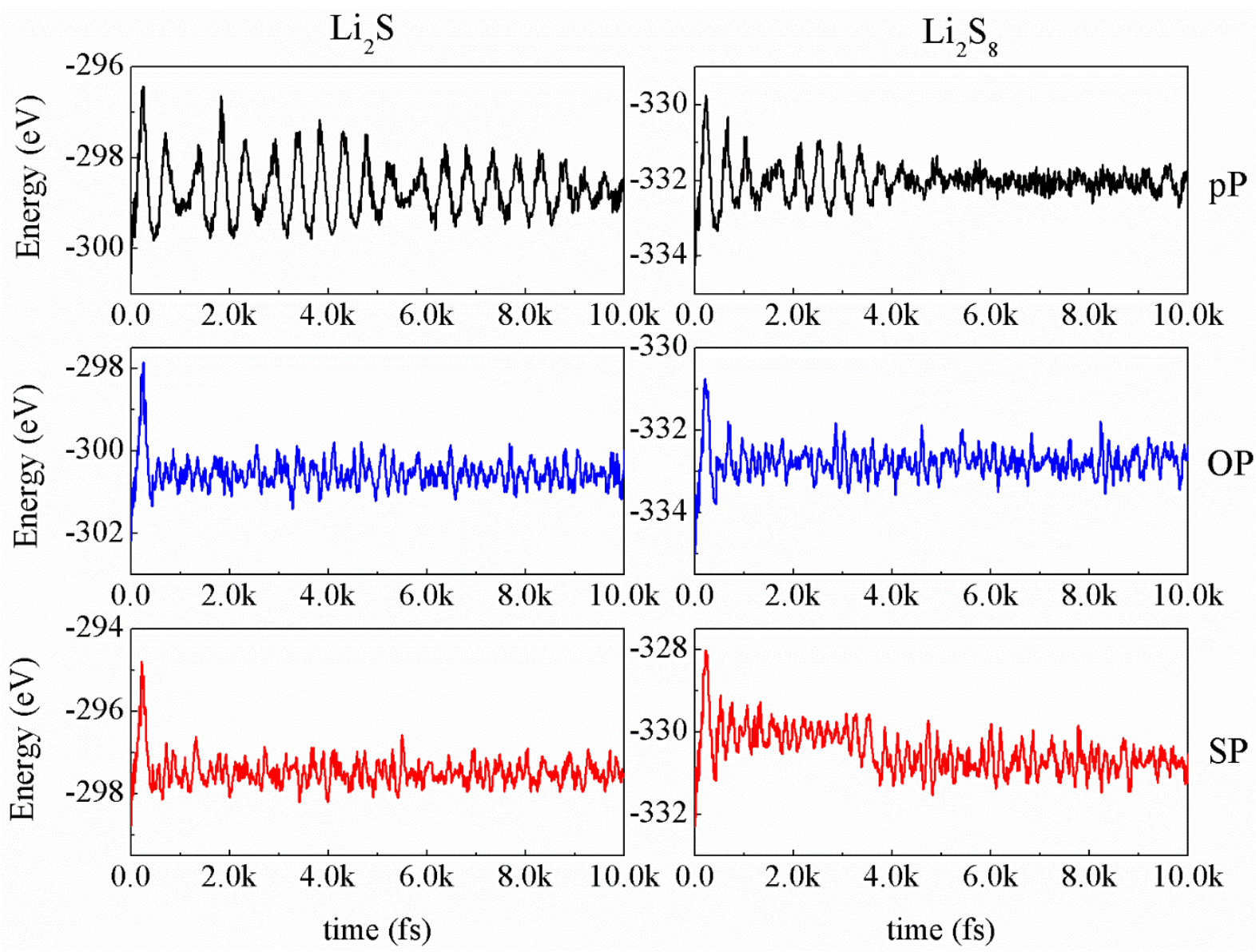
Fig. S12

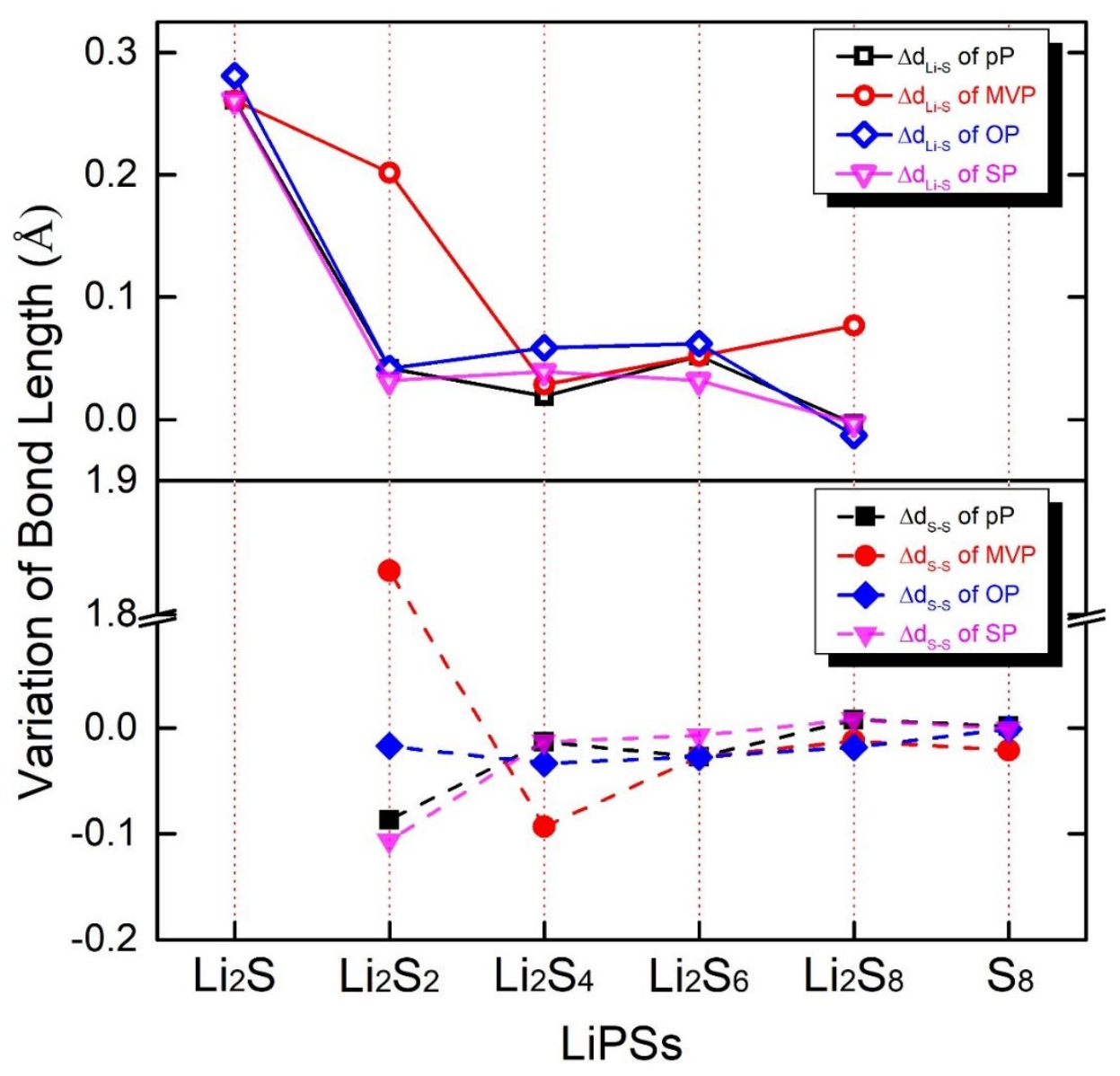

